\title{
The Enigma of the Pericardial Reaction in Rheumatic Heart Disease
}

\author{
S. Siew ${ }^{1}$ \\ 1. Division of Human Pathology, College of Osteopathic Medicine, Michigan State University, East \\ Lansing ,MI. 48824-1316
}

Rheumatic heart Disease is a pancarditis i.e. it affects all three layers of the heart-the endocardium, both valvular and parietal; the myocardium and the pericardium. Of the three layers, the pericardium shows the least stigmata in the chronic stage (Fig. 1), in spite of the extensive acute pericardial reaction in the first decade of life (Fig.2). Figure 1 shows isolated thickening of the visceral pericardium over the left atrial appendage. The patient was a 19 year old woman, who had had documented rheumatic heart disease since she was 9 years old. Such minimal involvement of the pericardium is surprising in view of the abundant acute fibrinous exudate, involving both the visceral and parietal pericardium- the so called "bread and butter" heart of the acute reaction-Figure 2. The patient was a 10 year old boy.

In an analysis, in South Africa, of 757 cases of Rheumatic Heart Disease in the 21 year period of 1936 to 1956, [1], when rheumatic heart disease was prevalent, gross involvement of the pericardium was observed in 39 cases:-fibrinous pericarditis-2;pericardial adhesions with circumscribed obliteration, particularly, near the apex-23; adhesive pericarditis with obliteration of the sac (synechia cordis)-8. "Adherent pericarditis, one of the more innocent rheumatic lesions, is of little importance" [2]; mediastinopericarditis-6. It is difficult to understand how such an abundant acute fibrinous exudate (Fig. 2) can leave so little trace of pericardial involvement in the chronic stage of the disease (Fig. 1). Apparently, there is an inhibition of organization and fibrosis of the acute reaction, and, it undergoes resolution.

We studied the histopathology of acute fibrinous pericarditis. The myocardium and endocardium of these cases manifested the typical lesions of acute and chronic rheumatic carditis. Underlying the dense fibrinous exudate, there was focal evidence of the ingrowth of parallel rows of newly formed, thin walled blood vessels, accompanied by fibroblasts-typical granulation tissue of the stage of organization But, a more prominent feature was the presence of thick walled, well-formed blood vessels, and, an infiltration of lymphoplasmacytic cells, both diffusely among the blood vessels (Fig.3), and in large focal aggregates (Fig.4). In spite of the florid amount of fibrinous exudate, there was a paucity of polymorphonuclear leukocytes.

The unusually rich vascularity of the lesion, leads one to suggest that it may cut down on the amount of fibrous tissue that would have been formed in the usual course of organization of acute lesions. The prominent presence of plasma cells may indicate the development of an immune reaction, with the presence of antibodies, which may have inhibited the formation of fibrous tissue. These two findings may be factors contributing to the enigma of the pericardial reaction in rheumatic heart disease. 


\section{References:}

[1] S Siew, An Investigation Into The Problem of Rheumatic Heart Disease With Special Reference to the Pulmonary Complications. Thesis accepted for the Degree of Medicine, University of the Witwatersrand. 1963.

[2] P Wood, Diseases of the Heart and Circulation, 2ed., 1958, p.352.

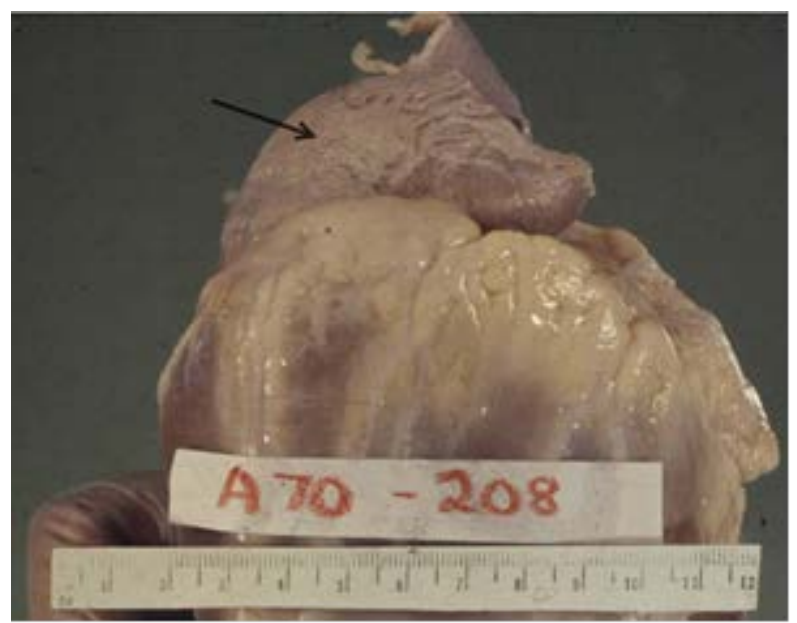

Figure 1. Focal thickening of pericardium over the left atrial appendage, female, 19 years old

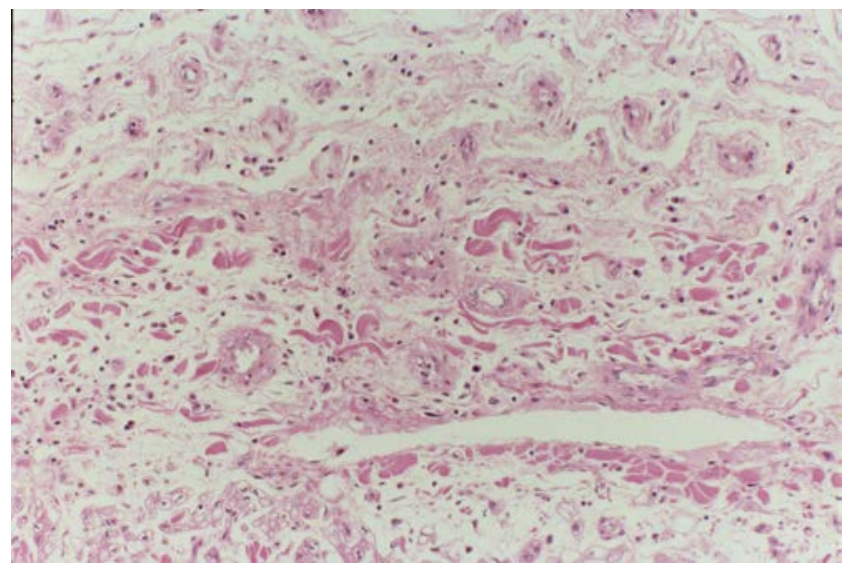

Figure 3. Rich vascularity and infiltration of plasma cells, original magnification 200x, female, 5 years old

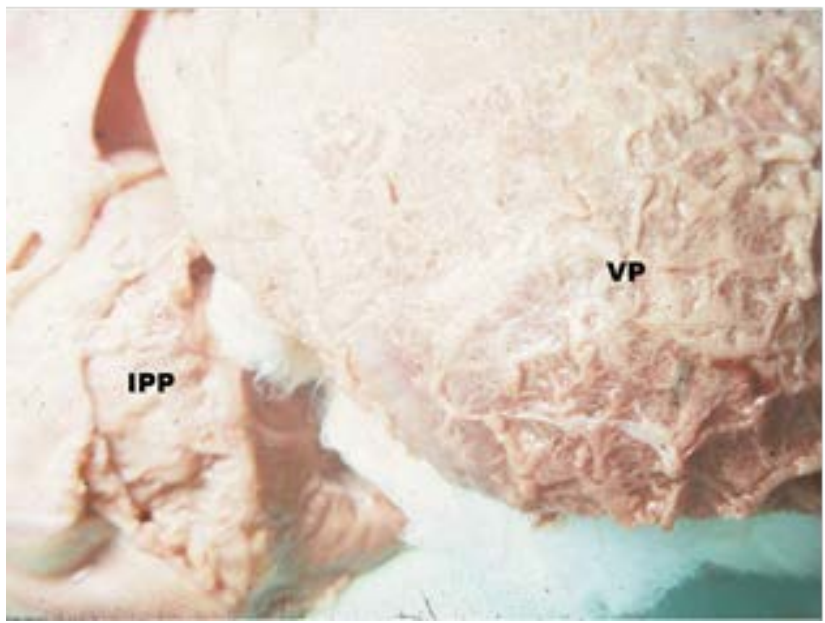

Figure 2. Acute fibrinous pericarditis - "Bread and Butter Heart", VP - Visceral pericardium; IPP - Inner layer parietal pericardium, male, 10 years old

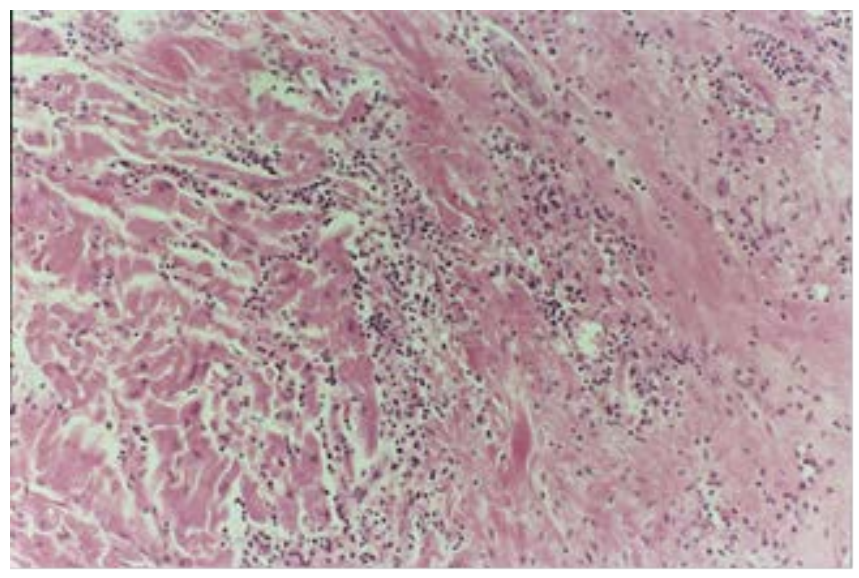

Figure 4. Aggregates of plasma cells, original magnification $200 \mathrm{x}$, female, 5 years old 